\title{
Nonuniform magnetization reversals in elliptical permalloy dots
}

\author{
Mei-Feng Lai ${ }^{\mathrm{a}}$, Zung-Hang Wei ${ }^{\mathrm{a}, *}$, Ching-Ray Chang ${ }^{\mathrm{a}}$, N.A. Usov ${ }^{\mathrm{b}}$, J.C. Wu ${ }^{\mathrm{c}}$, \\ Jun-Yang Lai ${ }^{\mathrm{a}}$ \\ a Department of Physics and Center for Nanostorage Research, National Taiwan University, Taipei, 10617, Taiwan \\ ${ }^{\mathrm{b}}$ Troitsk Institute for Innovation and Fusion Research, Troitsk, Moscow Region 142190, Russia \\ ${ }^{\mathrm{c}}$ Department of Physics, National Changhua University of Education, Changhua 500, Taiwan
}

Available online 3 May 2004

\begin{abstract}
Reversible and irreversible magnetization processes of the single-domain elliptical permalloy dot are studied by simulation. The magnetization curve of the reversible process obtained by applying the field along the hard axis of the elliptical dot is almost linear, as is the case in the single-domain ellipsoidal particle. Due to the occurrence of the non uniform reversal, the switching field is reduced significantly compared to that predicted by the Stoner-Wohlfarth model. The nucleation fields of elliptical dots are calculated as a function of ellipse's aspect ratio, and the function reveals a step-like behavior.
\end{abstract}

(C) 2004 Elsevier B.V. All rights reserved.

PACS: $75.60 . \mathrm{jk} ; 75.75 .+\mathrm{a}$

Keywords: Micromagnetic simulation; Stoner-Wohlfarth model; Magnetization reversal

Single-domain magnetic thin films are extensively used as unit cells in spintronic devices [1] such as, magnetic random access memory (MRAM). Above and below each cell electric currents pass through two perpendicular electrodes and generate a magnetic field, which can change the magnetization direction of the cell. Therefore, it is crucial to investigate the magnetization properties of patterned thin films to ensure high performance while accessing the devices. Here

\footnotetext{
*Corresponding author. Tel.: + 886-2-33665183; fax: + 8862-23639984.

E-mail address: zhwei@phys.ntu.edu.tw (Z.-H. Wei).
}

in this article we study the magnetization curves of the reversible and irreversible processes by applying fields along the hard and easy axes of the elements, respectively. The hysteresis loops of the irreversible processes with fields applied in various directions are also investigated. Besides, the switching fields of elliptical thin films as a function of the ellipse's aspect ratios are also studied.

The three-dimensional micromagnetic simulations of submicron-sized elliptical permalloy thin films are made by the time integration of the Landau-Lifschitz-Gilbert (LLG) equation [2,3]. In our simulation, we take into account the terms of magnetostatic interactions, exchange 
interactions, anisotropy field, and Zeeman field. The typical parameters of the soft-type permalloy films are as follows: exchange constant $C=2 A=2 \times 10^{-6} \mathrm{erg} / \mathrm{cm}$, and saturation magnetization $M_{\mathrm{s}}=800 \mathrm{emu} / \mathrm{cm}^{3}$. The uniaxial anisotropy constant $K_{1}$ is assumed to be $10^{3} \mathrm{erg} / \mathrm{cm}^{3}$ [4], which is actually too small to make any significant influence on the total energy. The element is discretized into many small cubic cells to approximate the ellipse. The size of the meshed cell is set to be lower than $7.5 \mathrm{~nm}$, which is smaller than the exchange length of the permalloy material [5], to assure the precision of our simulation. Our subdivision procedure produces a step-like boundary, so the surface magnetic pole density on the lateral side of the elements oscillates around the averaged pole density of an ideally smooth surface. Nevertheless, deviations in the order of several nanometers from a smooth lateral surface are also expected in the experiment [6]. When the largest magnetization angular variation between successive iterations is below $10^{-8}$, the system is assumed to reach the equilibrium state in our simulation.

A comparison of the magnetization process of the single-domain elliptical thin film with its corresponding Stoner-Wohlfarth [7] ellipsoid provides better understanding of the behaviors of the single-domain ellipse under magnetic field. The corresponding Stoner-Wohlfarth ellipsoid has the same volume as the elliptical thin film under investigation, and its demagnetization factors can be determined from the Brown-Morrish theorem [8]. Fig. 1 shows the simulation results of the magnetization curves of three sets of singledomain elliptical thin films and their corresponding Stoner-Wohlfarth ellipsoids when the magnetic fields are applied along the hard axes. Each magnetization curve of the single-domain ellipse is close to that of its corresponding ellipsoids; however, the magnetization rotation of the single-domain ellipse towards the magnetic field direction is not a coherent rotation due to the nonuniform demagnetization field inside the film. Besides, the magnetization of the elliptical thin film does not saturate under a finite magnetic field but only rotates gradually with the increasing field, as opposed to the case in the Stoner-Wohlfarth ellipsoid. Also note that the magnetization process

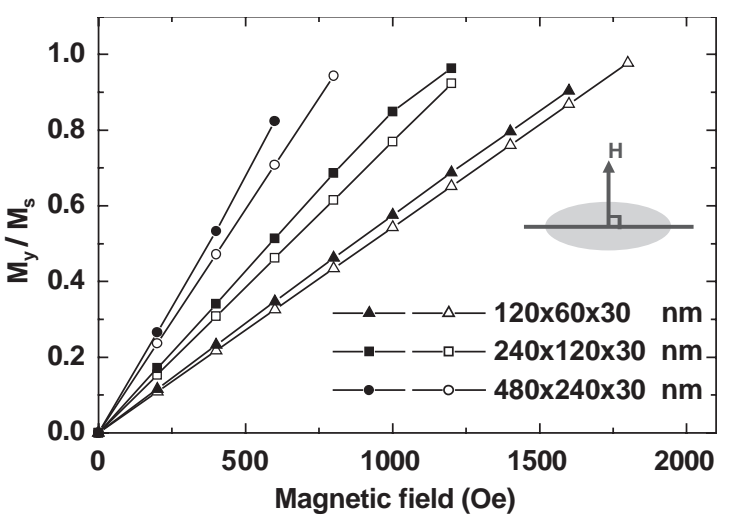

Fig. 1. Magnetization curves of the single-domain elliptical (with solid symbols) thin films and the corresponding StonerWohlfarth ellipsoidal (with open symbols) particles, which, respectively, have the same volumes as the three elliptical thin films, when a field $H$ is applied along the hard axis. The three sets of curves correspond to three different particles with dimensions indicated by long-axis $\times$ short-axis $\times$ thickness.

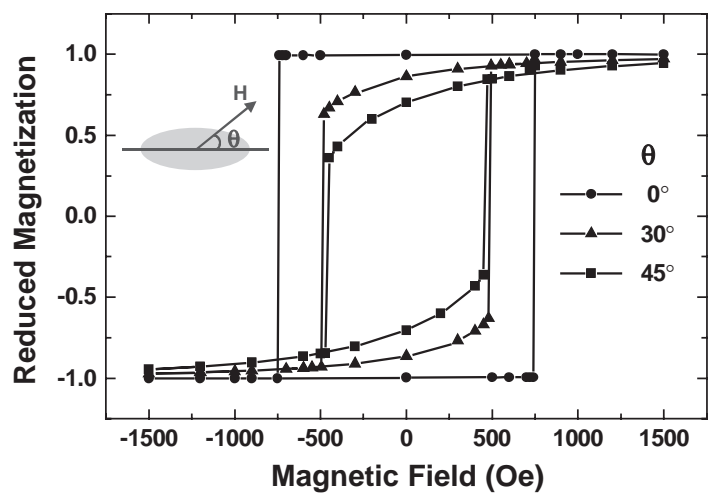

Fig. 2. Hysteresis loops of the permalloy elliptical thin film at various field angles $\theta$ with respect to the particle's long-axis. The dimension of the particle is $240 \mathrm{~nm}$ in long-axis, $120 \mathrm{~nm}$ in short-axis, and $30 \mathrm{~nm}$ in thickness.

is reversible, and the hysteresis is zero. For the irreversible processes, the simulation results of the hysteresis loops of the elliptical elements at different field angles $\left(0^{\circ}, 30^{\circ}\right.$, and $\left.45^{\circ}\right)$ with respect to the ellipse's long-axis are shown in Fig. 2. The hysteresis loops of the ellipse are almost rectangular at $0^{\circ}$ field angle, similar to the case in the ellipsoid. However, due to the nonuniform remagnetization process, the switching fields of the ellipses reduce considerably compared with 


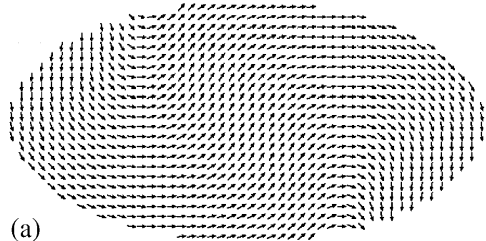

(a)

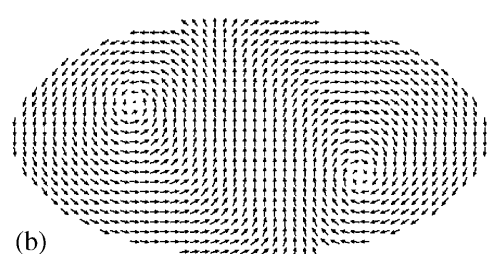

(b)

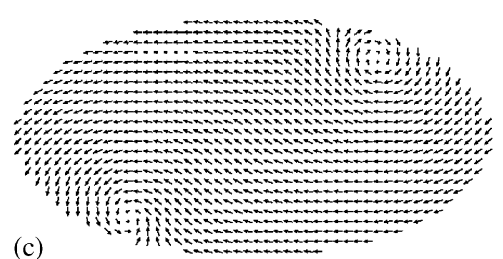

(c)

Fig. 3. Evolution of magnetization patterns during the nonuniform remagnetization process. The quasiuniform state initially directed to the right reverses to the left via the transient process (a) to (c). The dimension of the particle is the same as the one in Fig. 2.

Table 1

Switching fields of elliptical particles $\left(H_{\mathrm{c}}\right)$ and those of the corresponding Stoner-Wohlfarth ellipsoids $\left(H_{\mathrm{c}, \mathrm{SW}}\right)$ with different sizes and field angles

\begin{tabular}{llll}
\hline Dimensions $\left(\mathrm{nm}^{3}\right)$ & Field angle & $H_{\mathrm{c}, \mathrm{sw}}(\mathrm{Oe})$ & $H_{\mathrm{c}}(\mathrm{Oe})$ \\
\hline $240 \times 120 \times 30$ & $0^{\circ}$ & 1300.00 & 750 \\
& $30^{\circ}$ & 682.53 & 495 \\
$360 \times 120 \times 30$ & $45^{\circ}$ & 651.25 & 470 \\
& $0^{\circ}$ & 1787.20 & 1000 \\
$720 \times 240 \times 30$ & $30^{\circ}$ & 937.85 & 790 \\
& $45^{\circ}$ & 894.87 & 790 \\
& $0^{\circ}$ & 1149.00 & 520 \\
& $30^{\circ}$ & 603.46 & 440 \\
& $45^{\circ}$ & 575.80 & 440 \\
\hline
\end{tabular}

Dimensions shown in the first column are indicated by longaxis $\times$ short-axis $\times$ thickness.

those of the corresponding Stoner-Wohlfarth ellipsoids. The remagnetization process begins with the nucleation of several vortices at the boundary, as shown in Fig. 3. It is followed by the penetration of these vortices across the ellipse along the short-axis direction, and then ends up with the annihilation of the vortices at the boundary. As the magnetic fields are applied at different angles, the hysteresis loops are also similar to the ellipsoids and the switching fields are reduced significantly with respect to the corresponding Stoner-Wohlfarth ellipsoids as well.

The simulation results of the switching fields of the ellipses and the analytical results of their corresponding Stoner-Wohlfarth ellipsoids with different sizes at different field angles are shown in Table 1. For the ellipses, the switching fields are reduced when the applied fields deviate from the easy axis, as is the case in ellipsoids. At $30^{\circ}$ and $45^{\circ}$ field angles the switching fields are exactly the same in the ellipses with aspect ratio 3:1, i.e., 790 Oe for sample with dimension $360 \mathrm{~nm} \times$ $120 \mathrm{~nm} \times 30 \mathrm{~nm}$ and 440 Oe for sample with dimension $720 \mathrm{~nm} \times 240 \mathrm{~nm} \times 30 \mathrm{~nm}$; while for the ellipse with aspect ratio $2: 1$, i.e., $240 \mathrm{~nm} \times$ $120 \mathrm{~nm} \times 30 \mathrm{~nm}$, the switching fields are different at $30^{\circ}$ and $45^{\circ}$ field angles. This is due to the fact that vortices are more easily nucleated in the two ends of the long-axis with larger curvature when the total magnetization deviates from the easy axis direction towards the magnetic field direction and is bended along the boundary as an oblique field is applied. Since the switching occurs when the vortices are nucleated, the $30^{\circ}$ and $45^{\circ}$ field directions have the same nucleation field for the 3:1 case, in which the curvatures are larger at the two ends of the long-axis. However, vortices are not easily nucleated in the two ends of the longaxis with smaller curvature, so the field direction has more obvious influence on the nucleation fields.

Fig. 4 shows the simulation result of the nucleation field as a function of the ellipse's aspect ratio with fixed thickness $L_{z}=30 \mathrm{~nm}$ and shortaxis length $L_{y}=120 \mathrm{~nm}$. The nucleation field saturate as the aspect ratio is large enough. Due to the step interval of the applied fields adopted in the numerical simulation, the upper bound and lower bound of the applied field are provided here to locate the nucleation field. When the absolute value of the applied field is smaller than that of the upper bound, the single-domain state remains stable, but when the absolute value of the applied field is larger than that of the lower bound, the single-domain state is reversed. The behavior of saturation in the nucleation field is qualitatively in agreement with the experimental results obtained by Johnson et al. [9]. This result comes from the 


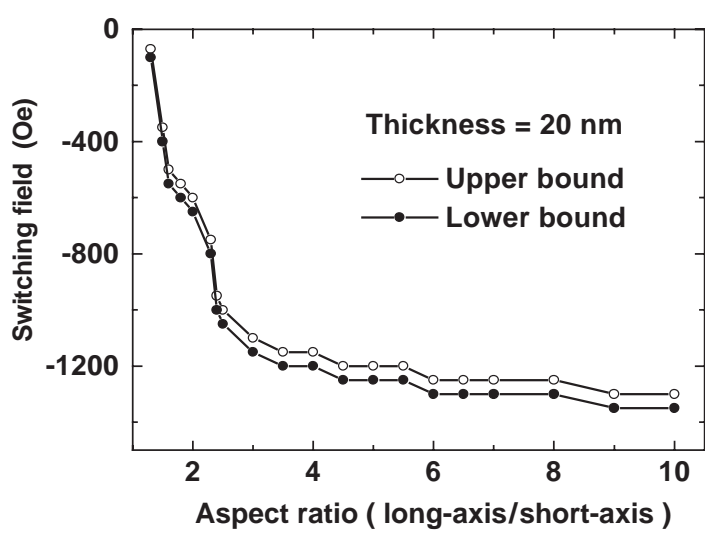

Fig. 4. Simulation result of the nucleation field of elliptical particles with thickness $L_{z}=20 \mathrm{~nm}$ and short-axis $L_{y}=100 \mathrm{~nm}$ as a function of the particle's aspect ratio. When the absolute value of the applied field is smaller than that of the upper bound, the quasiuniform state remains stable, but when the absolute value of the applied field is larger than that of the lower bound, the magnetization is reversed.

fact that near the middle part of the ellipse the demagnetization field caused by the two magnetic poles at the two ends of the long-axis reduces as the long-axis length increases, and the nucleation of the middle part is therefore mainly influenced by the external field.

This work was supported in part by the ROC National Science Council under Grant NSC 922112-M002-050 and the Technology Development Program for Academia under Grant 92-EC-17-A08-S1-0006.

\section{References}

[1] G.A. Prinz, Science 282 (1998) 1660.

[2] M.E. Schabes, J. Magn. Magn. Mater. 95 (1991) 249.

[3] N.A. Usov, et al., J. Magn. Magn. Mater. 130 (1994) 275.

[4] I.A. Campbell, et al., in:E.P. Wohlforth (Ed.) Ferromagnetic materials, Vol. 2, North-Holand, Amsterdam, The Netherlands. 1980.

[5] E.D. Boerner, et al., J. Appl. Phys. 87 (9) (2000) 5389.

[6] M. Herrmann, et al., J. Appl. Phys. 87 (6) (2000) 2994.

[7] E.S. Stoner, et al., Philos. Trans. R. Soc. London A 240 (1948) 599.

[8] W.F. Brown Jr., Micromagnetics, Interscience, NY, 1963.

[9] J.A. Johnson, et al., Appl. Phys. Lett. 77 (2000) 4410. 